\title{
Relato de experiência: a construção de um curso pré-vestibular popular vinculado ao curso de medicina
}

\author{
Experience report: the construction of a popular pre-university entrance course linked to \\ the medical course
}
Informe de experiencia: la construcción de un curso de acceso preuniversitario popular vinculado a la carrera de medicina

Lucas Vieira $^{1 *}$, Inauã Weirich Ribeiro ${ }^{1}$, Leonardo Rickes da Rosa1 ${ }^{1}$, Maria Isabel Lopes ${ }^{1}$, Guilherme Liberato da Silva ${ }^{1}$, Fernanda Rocha da Trindade ${ }^{1}$.

\begin{abstract}
RESUMO
Objetivo: Relatar a experiência da dinâmica de seleção e funcionamento de um curso pré-vestibular popular criado e desenvolvido como projeto de extensão por alunos de medicina de uma universidade comunitária do estado do Rio Grande do Sul. Relato de experiência: Estudantes da graduação estruturaram um curso prévestibular e desenvolveram um modelo próprio de seleção, baseado em experiências prévias com processos seletivos e nas Multiple Mini-Interviews (MMI), o qual une a avaliação teórica (primeira etapa) com cenários temáticos (segunda etapa). A seleção visa abranger não somente competências cognitivas. $A$ avaliação teórica evidenciou que os candidatos apresentaram dificuldades em matemática, química e física. Já os cenários temáticos contribuíram na identificação de candidatos que expressassem traços de trabalho em equipe, empatia, motivação e interesse em participar do projeto. Após o processo, o projeto se desenvolveu com aulas e simulados ao longo de um ano letivo. Considerações finais: A incorporação dos cenários temáticos contribuiu na redução das desistências ao longo do ano, à medida em que a avaliação teórica possibilitou o conhecimento das principais dificuldades dos candidatos. Portanto, a seleção, composta pelas duas etapas, coadunaram com as particularidades do projeto de extensão e auxiliaram na aproximação e manutenção dos alunos-acadêmicos da realidade regional em que a universidade está inserida.
\end{abstract}

Palavras-chave: Extensão, Ensino superior, Educação popular.

\begin{abstract}
Objective: to present an experience of the dynamics of selection and operation of a popular pre-university entrance course created and developed as an extension project by medical students from a community university in the state of Rio Grande do Sul. Experience report: Undergraduate students structured a preuniversity course and developed their own selection model, based on previous experiences with selection processes and in the Multiple Mini-interviews (MMI), which is a theoretical evaluation (first stage) with thematic scenarios (second stage). Visa selection encompasses not only cognitive skills. The theoretical evaluation evidences that students dissipate in mathematics, chemistry and physics. The thematic scenarios, on the other hand, contributed to the identification of candidates who expressed traits of teamwork, empathy, motivation and interest in participating in the project. After the process, the project develops with classes and simulations over an academic year. Final considerations: An incorporation of thematic scenarios contributed to the reduction of dropouts throughout the year, as the theoretical evaluation enabled the knowledge of the main difficulties of the candidates. Therefore, a selection, made up of the two stages, was in keeping with the particularities of the extension project and assisting in the approximation and maintenance of studentsundergraduates students from the regional reality in which the university is inserted.
\end{abstract}

Key words: Extension, University education, Popular education.

1 Universidade do Vale do Taquari (Univates), Lajeado - RS. *E-mail: lucas.vieira@universo.univates.br SUBMETIDO EM: $3 / 2021 \quad$ ACEITO EM: 3/2021 | PUBLICADO EM: 4/2021 


\section{RESUMEN}

Objetivo: Informar la experiencia de la dinámica de selección y operación de un curso preuniversitario popular creado y desarrollado como un proyecto de extensión por estudiantes de medicina de una universidad comunitaria en el estado de Rio Grande do Sul. Informe de experiencia: Estudiantes de pregrado estructuraron un curso preuniversitario y desarrollaron su propio modelo de selección, basado en experiencias previas con procesos de selección y en Mini-Entrevistas Múltiples (MMI), que une la evaluación teórica (primera etapa) con escenarios temáticos (segunda etapa). La selección tiene como objetivo cubrir no solo las habilidades cognitivas. La evaluación teórica mostró que los candidatos tenían dificultades en matemáticas, química y física. Los escenarios temáticos, por su parte, contribuyeron a la identificación de candidatos que expresaron rasgos de trabajo en equipo, empatía, motivación e interés por participar en el proyecto. Tras el proceso, el proyecto se desarrolló con clases y simulaciones durante un curso académico. Consideraciones finales: La incorporación de escenarios temáticos contribuyó a la reducción de la deserción escolar a lo largo del año, ya que la evaluación teórica permitió conocer las principales dificultades de los candidatos. Por tanto, la selección, compuesta por las dos etapas, fue acorde con las particularidades del proyecto de extensión y ayudó a acercar a los estudiantes a la realidad regional en la que se ubica la universidad.

Palabras clave: Extensión, Enseñanza superior, Educación popular.

\section{INTRODUÇÃO}

Em 2018, o número de matrículas na educação superior atingiu a marca de 8,45 milhões de alunos, constituindo 3,4 milhões em cursos de graduação, sendo 83,1\% em instituições privadas (INEP, 2019). Mesmo com o significativo aumento das matrículas, sabe-se que cerca de 69,5 milhões de pessoas com 25 anos ou mais não possuem o ensino médio completo (IBGE, 2019). A rede pública de ensino é responsável por cerca de $87,4 \%$ dos alunos de ensino médio do país, enquanto a rede privada é a origem de $73,7 \%$ dos estudantes de graduação (IBGE, 2019). Evidencia-se, portanto, a desigualdade de acesso ao ensino superior no Brasil quando comparada à origem escolar dos alunos de escolas públicas e privadas no Brasil (CARVALHO MM e WALTENBERG FD, 2015). Em decorrência desse cenário de desigualdade no acesso à universidade, o número de estudantes da rede pública que acessam a graduação é restrito (SALATA A, 2018).

É notório, no contexto de ensino básico e superior, as adversidades de ensino e aprendizagem no Brasil (DIAZ MDM, 2012). O maior estudo sobre educação do mundo, o Programa Internacional de Avaliação de Estudantes (PISA), apontou que o Brasil tem baixa proficiência em leitura, matemática e ciências, quando comparado com outros 78 países participantes da análise. A edição de 2018 revelou que $68,1 \%$ dos estudantes brasileiros, com 15 anos de idade, não possuem nível básico de matemática; em ciências, o número chega a 55\%; em leitura, 50\%; os índices estão estagnados desde 2009 (INEP, 2019). Tal resultado indica desafios à educação brasileira, não só em relação ao avanço nos estudos, como também na busca por melhores oportunidades no mercado de trabalho e na participação social.

Diante desse cenário de desigualdade, surgiram diversos cursinhos pré-vestibulares populares, principalmente na década de 2000, sendo, muitos deles, desenvolvidos por universitários voluntários que ministram aulas para estudantes oriundos, em sua maioria, do ensino público e que almejam acessar o ensino superior (KATO SD, 2011; WHITAKER DCA, 2010). No Brasil, na cidade de São Paulo, diversos projetos buscam a construção desses espaços, objetivando a transformação da realidade através da educação popular pluricultural (SORIANO LA, et al. 2016). Outros cursinhos estão presentes em outras cidades do país, muitos vinculados às universidades, principalmente públicas (CARVALHO MF e FREITAS MC, 2013).

Em consonância com essa realidade, neste trabalho é apresentado a experiência de um cursinho popular pré-vestibular que foi criado e é desenvolvido em meio a uma universidade comunitária no interior do Estado do Rio Grande do Sul, na cidade de Lajeado/RS. Tem-se como objetivo apresentar a dinâmica de seleção e funcionamento de um cursinho popular pré-vestibular que funciona como um projeto de extensão. O projeto 
foi criado por estudantes do curso de medicina, com o objetivo de preparar anualmente estudantes da rede pública de ensino para ingresso no ensino superior.

\section{RELATO DE EXPERIÊNCIA}

O Cursinho Popular da Univates (VestVates) foi criado em 2017 e surgiu da união de estudantes do curso de medicina, visando atingir estudantes do terceiro ano da rede pública de ensino médio ou concluintes do ciclo básico de educação que não teriam condições sociais e econômicas para ingressar em um cursinho prévestibular privado. $O$ projeto é organizado pelos estudantes, que formam a coordenação discente, e coordenado por professores da universidade, possuindo como trabalho focal é voltado para o Exame Nacional do Ensino Médio (ENEM). Através desse dispositivo, os alunos atendidos disputam uma vaga para ingresso no ensino superior tanto na esfera pública via Sistema de Seleção Unificada (SISU) e vestibulares tradicionais, quanto na esfera privada via Fundo de Financiamento ao Estudante do Ensino Superior (FIES), Programa Universidade para Todos (ProUni) ou outros sistemas de ingresso.

O processo seletivo tem por comunidade foco as pessoas da rede pública de ensino. Esse é realizado a partir de uma prova cognitiva, como ocorre em inúmeros processos vestibulares brasileiros, e, após, uma avaliação não cognitiva. Para participar do processo de seleção, a pessoa apresentou certificado, caso tivesse concluído o ensino médio, ou certidão de matrícula, desde que cursando o $3^{\circ}$ ano, em rede pública de ensino. O processo de seleção, composto por 2 etapas, foi construído idealizando a seleção de pessoas que demonstrem interesse e comprometimento com a proposta do projeto, portanto a etapa cognitiva possui um menor peso na nota final. A prova cognitiva (primeira fase da seleção), elaborada pelos voluntários e coordenação do projeto, foi constituída por 50 questões de múltipla escolha, com 5 assertivas, abrangendo todas as disciplinas obrigatórias do ensino médio, com peso de $25 \%$. Os cenários temáticos foram a segunda fase da seleção, com peso de $75 \%$. O somatório das notas totalizou o desempenho do candidato no processo seletivo.

A primeira parte do processo seletivo foi construída com finalidade de identificar os conhecimentos prévios dos candidatos. No ano de 2020, 45 candidatos participaram da prova cognitiva, obtendo-se a média de 14,64 acertos, sendo 24 acertos a maior pontuação obtida. Os menores índices de acertos foram observados nas disciplinas de matemática, química e física. Já os cenários temáticos (segunda fase da seleção) buscaram observar a empatia, pensamento crítico, trabalho em equipe, liderança, ética, compaixão e motivação dos candidatos. Isso porque, ao longo do ano letivo, foi fundamental que os alunos participantes assumissem responsabilidades em relação aos seus processos de aprendizagem, de modo que a coordenação do curso constantemente enviasse feedbacks buscando acompanhamento individual conforme as demandas dos alunos. Nesta etapa, houve 4 ausências, portanto, 41 candidatos que realizaram a prova participaram.

Com o intuito de aprimorar a seleção realizada nos anos anteriores, foi desenvolvida e incluída a avaliação não cognitiva, por meio de aplicação de cenários temáticos. Tal processo teve por objetivo avaliar competências denominadas não cognitivas e selecionar pessoas que conseguissem trabalhar em equipe e que tivessem comprometimento com o projeto. Os cenários temáticos consistiram em uma série estruturada de 4 estações de avaliação com tempo controlado. Cada estação foi composta por resolução de desafios que deram aos candidatos diferentes oportunidades de expor suas impressões e habilidades. A base para a elaboração dos cenários temáticos foram as Multiple Mini-Interviews (MMI) (SAYS FE, et al., 2013; EVA KW, et al., 2019). A proposta das MMI pautou-se em avaliações padronizadas e abordagens de amostra múltipla, a fim de avaliar a ampla competência da pessoa e evidenciar pontos fortes e fracos do participante. Com esse referencial, as estações do processo seletivo do Cursinho foram desenvolvidas e adaptadas pela coordenação do projeto de extensão.

Em cada estação, o candidato foi avaliado objetivamente com uma nota de 0-10 em cada um dos eixos, conforme a Tabela 1. A análise objetiva se deu pela necessidade de diminuir os níveis de variações nas pontuações causadas pela subjetividade do entrevistador (ROBERTS C, et al., 2010; CALLWOOD A, et al., 2018). 
Tabela 1 - Caracterização dos cenários e itens avaliados em cada estação.

\begin{tabular}{ll}
\hline Estação & \multicolumn{1}{c}{ Competências e habilidades avaliadas } \\
\hline - Menino do dedo verde & $\begin{array}{l}\text { Relação teoria-prática nas proposições das atividades/intervenções } \\
\text { Criatividade (o quanto o candidato consegue criar e imaginar) } \\
\text { Argumentação (o quanto o candidato consegue persuadir com a sua história) }\end{array}$ \\
\hline $\begin{array}{l}\text { Capacidade de trabalhar em equipe (atitude, senso crítico e decisão) } \\
\text { (Flores) }\end{array}$ & $\begin{array}{l}\text { Identifica e contextualiza situações (como o candidato percebe e identifica a } \\
\text { flor em relação ao projeto) } \\
\text { Intervenção (propor formas de intervenção do contexto explorado de acordo } \\
\text { com as demandas). }\end{array}$ \\
\hline 3 - Balões & $\begin{array}{l}\text { Reflexão e mudança (criar estratégias de inserção e implicação no espaço) } \\
\text { Acolhimento e vínculo (relacionar-se com o outro e formação de vínculos) } \\
\text { Postura ética (como o candidato se comporta com os demais) }\end{array}$ \\
\hline 4 - Escrita da carta de & $\begin{array}{l}\text { Capacidade de argumentação } \\
\text { Norma culta da língua portuguesa } \\
\text { intenção }\end{array}$ \\
\hline
\end{tabular}

Fonte: Vieira L, et. al., 2021.

A estação 1 aplicou atividades que relacionaram teoria e prática, de modo que a criatividade e a argumentação do candidato podiam ser expressas. Para isso, foi selecionada uma adaptação do conto "O menino do Dedo Verde" de Maurice Druon (1989), pois é uma leitura de fácil entendimento e permeada de reflexões acerca das relações humanas com a educação. $O$ candidato tinha 2 minutos para ler a adequação e, ao finalizar, relacionar o texto com o entendimento prévio que tinha sobre o projeto. A nota média dessa estação foi de 6,14 , com variações significativas de repertório, foi percebida uma evidente diferença na capacidade argumentativa e de interpretação entre os candidatos.

Com o intuito de averiguar as atitudes, o senso crítico e tomada de decisões, na estação 2 o candidato precisou identificar e contextualizar situações e sugerir maneiras de intervir. Para isso, os participantes foram divididos em pequenos grupos de 3 e precisaram escolher entre flores dispostas em uma mesa. Cada flor representou uma qualidade e o grupo necessitou registrar em um cartaz as palavras que a representam. Após 2 minutos, o avaliador ordenou que os candidatos trocassem de lugar e que os grupos ajudassem na elaboração dos cartazes dos demais grupos. Para tal fim, foi selecionado um líder do grupo que apresentou o cartaz para os demais. Essa foi a estação com a maior nota média, com 7,58. A maioria dos candidatos trabalharam em coletivo, elaborando apontamentos e sugestões positivas nos cartazes dos demais grupos. Observou-se discreta dificuldade em algumas pessoas assumirem o cargo de líder para apresentar o trabalho.

Além disso, na estação 3 foram avaliadas a criatividade de inserção e implicação em um determinado espaço, assim como o acolhimento, a criação de vínculos e a postura ética dos candidatos. O cenário iniciou com a distribuição de balões vazios, com as seguintes palavras: lealdade, respeito, trabalho, medo, honestidade, paciência, compromisso, ambição, inveja e preguiça. Com o balão selecionado, formaram um círculo em que, após encherem os balões, jogaram para cima sem os deixar cair. Em determinado momento, parte do grupo foi convidado a se sentar, deixando seu balão para que outra parte do grupo evitasse a queda dos demais balões, no intuito de avaliar o espírito de trabalho em equipe. Quando restavam apenas 5 participantes, os colegas sentados voltaram à dinâmica e retomaram a posse de seu balão, e finalizaram a etapa estourando-o, pegando a palavra e realizando uma rápida fala a respeito do significado. Nesse cenário, os candidatos entenderam a dinâmica e obtiveram bons resultados, com a média de 7,54. Os avaliadores pontuaram notas semelhantes para os candidatos.

Para finalizar o percurso dos cenários temáticos, a estação 4 avaliou a argumentação dos candidatos, por meio da norma culta da língua portuguesa e a intenção pessoal. Os candidatos elaboraram um texto dissertativo-argumentativo, em até 10 linhas, em modalidade escrita formal da língua portuguesa. $O$ tema foi apresentado com uma pergunta: "Por que quero ser do VestVates?". O participante selecionou, relacionou e organizou, de forma coerente e coesa, as informações, fatos e opiniões para defesa do seu ponto de 
vista. Percebeu-se que esse cenário foi o qual os candidatos tiveram mais dificuldades, resultando na menor nota média da segunda etapa, com 5,96. Tal nota se justifica pelos inúmeros erros gramaticais encontrados nos textos e, também, pela inconsistência dos argumentos utilizados na construção do trecho.

Depois da divulgação dos resultados, do processo de matrícula e aluna inaugural, os alunos selecionados frequentaram o projeto durante 10 meses, com aulas ministradas de segunda a sexta-feira, das $19 \mathrm{~h} 10$ às 22h30. Foram 3 aulas de 60 minutos por dia, com um intervalo de 10 minutos entre a segunda e a terceira aula. A organização de 3 horas por dia de aula foi resultado de um longo debate realizado com os alunos que frequentaram o projeto no ano de 2019, visto que a grade era composta por 5 aulas de 40 minutos. O projeto, ao longo do ano de 2020, mesmo com suas atividades virtualizadas de forma síncrona, teve 8 desistências $(26,6 \%)$. Isso pode estar relacionado com os efeitos da pandemia da COVID-19. Tal índice, ainda que tenha ocorrido em um ano letivo totalmente atípico, foi menor que no ano anterior; em 2019, houve 12 desistências (40\%).

É importante destacar que a grade curricular do projeto, conforme Tabela 2, foi dividida por eixos, de acordo com as diretrizes e grandes áreas do ENEM: Matemática e suas tecnologias, Ciências Humanas e suas tecnologias, Linguagens, códigos e suas tecnologias e Ciências da natureza e suas tecnologias. Cada eixo possui um conjunto de disciplinas e, no VestVates, elas são denominadas de frentes.

Tabela 2 - Matriz curricular do VestVates de 2019/2020/2021.

\begin{tabular}{|c|c|c|c|}
\hline $\begin{array}{l}\text { Componente } \\
\text { curricular }\end{array}$ & Frente & $\begin{array}{l}\text { Número de voluntários } \\
\text { (tutores) }\end{array}$ & $\begin{array}{c}\text { Número de voluntários } \\
\text { (monitores) }\end{array}$ \\
\hline Redação & Única & 1 & 10 \\
\hline Interpretação de texto & Única & 1 & 1 \\
\hline Literatura & Única & 1 & - \\
\hline Matemática & 1,2 e 3 & 3 & 3 \\
\hline História & 1 e 2 & 2 & 1 \\
\hline Geografia & 1 e 2 & 2 & 1 \\
\hline Biologia & 1,2 e 3 & 4 & 2 \\
\hline Física & 1,2 e 3 & 3 & 2 \\
\hline Química & 1,2 e 3 & 3 & 2 \\
\hline Sociologia & Única & 1 & 1 \\
\hline Sociologia & Única & 1 & 1 \\
\hline Língua Inglesa & Única & 1 & 1 \\
\hline Total & & 23 & 25 \\
\hline
\end{tabular}

Fonte: Vieira L, et. al., 2021.

\section{DISCUSSÃO}

Há, evidentemente, uma complementação de interesses na essência da extensão (FLORIANO MDP, et al., 2017). De um lado a instituição de ensino, com a teoria e a técnica, e no outro a comunidade, que faz a essência da instituição se manter dinâmica, fluída e capaz de formar além de profissionais habilitados cognitivamente, empáticos e responsáveis pela construção de uma sociedade mais igualitária (MEDEIROS MM, 2017). O projeto de extensão VestVates desenvolve a essência clássica da relação da extensão da universidade com a comunidade. Os acadêmicos dos diversos cursos de graduação, especialmente da medicina e licenciaturas, são atores centrais na condução do projeto. O estudante é confrontado e se permite vivenciar novos cenários, além dos propostos na grade curricular de seu curso, somando experiências na sua formação enquanto pessoa e profissional (FLORIANO MDP, et al., 2017).

Ainda, diante do cenário de escolarização dificultada, além do contexto de precarização estrutural da educação no Brasil, a extensão universitária surge como opção para inserção da comunidade acadêmica junto à sociedade. No VestVates, desde seu início, foram aplicadas provas cognitivas e análise socioeconômica como forma de seleção dos estudantes, visto que o projeto consegue atender, em seu atual 
formato, 30 alunos. Em decorrência da evasão escolar dos estudantes selecionados, compreendeu-se a necessidade de avaliar o engajamento com a proposta do curso, sendo acrescentada na seleção a avaliação não cognitiva, cujo um dos objetivos visa identificar o comprometimento da pessoa.

Após a reestruturação do processo seletivo, com a retirada da análise socioeconômica, criou-se os cenários temáticos, estruturados e pensados no intuito de avaliar habilidades não cognitivas, buscando diminuir os índices de evasão escolar e aumentar o engajamento dos estudantes junto às atividades propostas pelo projeto. Já a padronização do processo de seleção pela pontuação objetiva (0-10) se dá pela necessidade de diminuir a vulnerabilidade do candidato frente ao entrevistador, principalmente pela posição de sujeito como, por exemplo, étnico, raça e condição socioeconômica (CALLWOOD A, et al., 2018).

O plano de estudos tem a temporalidade de um ano, de maneira que é caracterizado pela revisão do conteúdo curricular do ensino médio já trabalhados, e não pela apresentação de novos conteúdos. Assim, a prova cognitiva tornou-se necessária, pois identifica os conhecimentos prévios dos candidatos para melhor organizar o planejamento pedagógico, com o cuidado de constituir-se menor peso no resultado (MOREIRA AA, 2017; SILVA FS, et al., 2020).

A construção da organização curricular do projeto, elaborada em frentes de trabalho, juntamente com a seleção de estudantes estruturada em duas etapas (cognitiva e não cognitiva), permite que o projeto tenha uma avaliação global do estudante, acompanhando-o em toda sua trajetória e, ao longo dessa, desenvolvendo intervenções pedagógicas que possam contribuir para a formação discente.

\section{CONSIDERAÇÕES FINAIS}

A seleção utiliza procedimentos padronizados de cunho cognitivo e não cognitivo para viabilizar um melhor atendimento aos estudantes da rede pública de ensino que ingressaram no projeto. A incorporação dos cenários temáticos contribuiu no processo seletivo de forma satisfatória, auxiliando no recrutamento de candidatos que tivessem interesse, motivação e entendimento do projeto, diminuindo o número de desistências ao longo do ano. Além disso, a matriz curricular contribui para o propósito do projeto e pode inspirar a elaboração e abordagem de plano de estudos para demais cursinhos populares. O número de participantes do seletivo, bem como a restrição do número de vagas do projeto, limitam a análise do impacto das ferramentas adotadas na seleção, mas não inviabilizam os apontamentos apresentados.

\section{AGRADECIMENTOS E FINANCIAMENTO}

Os autores agradecem à Universidade do Vale do Taquari (Univates) pelo apoio financeiro conferido no edital de extensão da instituição (edital no 086/2019-Reitoria/Univates), bem como a disponibilização do material necessário para a realização deste estudo.

\section{REFERÊNCIAS}

1. CARVALHO MM, WALTENBERG FD. Desigualdade de oportunidades no acesso ao ensino superior no Brasil: uma comparação entre 2003 e 2013. Econ. Apl, 2015; 19(2):369-396.

2. CARVALHO MF, FREITAS MC. Perspectivas e desafios dos cursinhos populares da Zona da Mata Mineira. Diálogos em Extensão, 2013; 02(01):172-182.

3. CALLWOOD A, et al. Personal domains assessed in multiple mini interviews (MMls) for healthcare student selection: A narrative synthesis systematic review. Nurse Educ Today, 2018; 64:56-64.

4. DIAZ MDM. (Des)igualdades de oportunidades no ensino médio brasileiro: escolas públicas e privadas. Economia: revista da ANPEC, 2012; 13(3a):553-568.

5. EVA KW, et al. Twelve tips for constructing a multiple mini-interview. Med Teach, 2019; 41(5):510-516.

6. FLORIANO MDP, et al. Extensão universitária: a percepção de acadêmicos de uma universidade federal do estado do Rio Grande do Sul. Revista Em Extensão, 2017;16(1):9-35.

7. IBGE. Pesquisa Nacional por Amostra de Domicílios (PNAD): Resultados Nacionais - PNAD 2018. Brasília: IBGE, 2019.

8. INEP. 2019. In: Censo da Educação Superior 2018: notas estatísticas. DEEB/INEP/MEC. Disponível em: https://download.inep.gov.br/educacao_superior/censo_superior/documentos/2019/censo_da_educacao_superior_2 018-notas_estatisticas.pdf. Acesso em: 11 jan. 2021. 
9. KATO SD. O papel dos cursinhos populares nos acessos e mudanças de perspectivas de seus participantes. Cadernos CIMEAC, 2011; 01:5-24.

10. MEDEIROS MM. A extensão universitária no Brasil - um percurso histórico. Revista Barbaquá, 2017; $1(1): 9-16$.

11. MOREIRA AA. Aprendizaje significativo como un referente para la organización de la enseñanza. Archivos de Ciencias de la Educación, 2017; 11(12).

12. ROBERTS $\mathrm{C}$, et al. Should candidate scores be adjusted for interviewer stringency or leniency in the multiple miniinterview?. Medical Education, 2010; 44(7):690-698.

13. SALATA A. Ensino Superior no Brasil das últimas décadas: redução nas desigualdades de acesso?. Tempo soc, 2018; 30(2):219-253.

14. SAYS FE, et al. Experience of establishment of multiple mini structure interview as part of student admission policy at Faculty of Medicine, King Abdulaziz University, 2011-2012. Medical Teacher, 2013; 35 (1):74-77.

15. SILVA FS, et al. Militarização e escola sem partido: repercussões no projeto político-pedagógico das escolas. Revista Exitus, 2020,10:01-26.

16. SORIANO LA, et al. Da saúde à extensão universitária: cursinho popular do PET-medicina, um projeto bem-sucedido na Faculdade de Medicina de Ribeirão Preto. Medicina (Ribeirão Preto), 2016;49(4):388-392.

17. WHITAKER DCA. Da "invenção" do vestibular aos cursinhos populares: um desafio para a Orientação Profissional. Rev. bras. orientac. prof, 2010;11(2):289-297. 